July 20, 2015

\title{
Denatured State Aggregation Parameters Derived from Concentration Dependence of Protein Stability
}

\author{
Arne Schön ${ }^{\S}$, Benjamin R Clarkson ${ }^{\S}$, Rogelio Siles ${ }^{\S}$, Patrick Ross", Richard \\ K Brown" and Ernesto Freire ${ }^{\S \llbracket \star}$ \\ ${ }^{\S}$ Department of Biology \\ Johns Hopkins University \\ 3400 North Charles \\ Baltimore, MD 21218 \\ ๆ AVIA Biosystems \\ 10E Commerce Way \\ Norton, MA 02766
}

Short Title: Structural Stability and Aggregation of Proteins

*To whom correspondence should be addressed at ef@jhu.edu 


\begin{abstract}
Protein aggregation is a major issue affecting the long term stability of protein preparations. Proteins exist in equilibrium between the native and denatured or partially denatured conformations. Often denatured or partially denatured conformations are prone to aggregate because they expose to solvent the hydrophobic core of the protein. The aggregation of denatured protein gradually shifts the protein equilibrium towards increasing amounts of denatured and ultimately aggregated protein. Recognizing and quantitating the presence of denatured protein and its aggregation at the earliest possible time will bring enormous benefits to the identification and selection of optimal solvent conditions or the engineering of proteins with the best stability/aggregation profile. In this paper a new approach that allows simultaneous determination of structural stability and the amount of denatured and aggregated protein is presented. This approach is based upon the analysis of the concentration dependence of the Gibbs energy $(\Delta G)$ of protein stability. It is shown that three important quantities can be evaluated simultaneously: 1) The population of denatured protein; 2) The population of aggregated protein; and, 3) The fraction of denatured protein that is aggregated.
\end{abstract}




\section{Introduction}

The identification of conditions that maximize the structural stability of the native state of proteins and prevent aggregation or other undesirable processes is essential for the development of protein therapeutics, protein formulations, process development conditions, quality control as well as basic protein sciences [1-4]. In general, there are two situations in which the capability of identifying aggregation tendencies is critical: 1) The engineering or selection of the mutations or protein variants that exhibit the best stability/aggregation profile; and,

2) The identification of the solution (formulation) conditions that maximize conformational stability and minimize aggregation. Long term stability of protein solutions is usually hampered by the presence of protein aggregates. Different mechanisms and kinetic processes account for the formation of these aggregates (see $[5,6]$ for recent publications). Among the potential sources of aggregates is the presence of denatured protein, which is the subject of this paper. Even if the amount of denatured protein is very small soon after a protein solution is prepared, it may have a tendency to aggregate, acting as a sink that eventually depletes the native state protein. This process has been often described in terms of the Lumry Eyring model [7] or variants of this model [8]. In protein therapeutics, aggregation does not only diminish the amount of biologically active protein but can also trigger undesirable immune responses and other adverse effects [9]. Identifying the best protein variant or the best formulation requires the ability to measure both the concentration of denatured protein and the fraction of 
that protein that is aggregated in a timely fashion. Using this information, the protein clone or the formulation with the optimal stability and aggregation attributes can be rapidly determined.

Direct measurement of the population of denatured protein immediately after a formulation or protein solution is prepared is extremely difficult. The population of denatured protein in a fresh formulation is exceedingly small (usually less than $0.01 \%$ of the total protein) which escape detection by conventional techniques. Commonly used accelerated stability or forced degradation assays essentially drive the denatured and aggregated population to measurable levels. An alternative method to determine the population of denatured protein relies on measurements of the structural equilibrium between native and denatured conformations. Determination of the Gibbs energy of stability $\left(\Delta G^{\circ}\right)$ allows calculation of the amount of denatured protein in a solution to the required levels $(<<0.01 \%$ of the total protein). Furthermore, since aggregation and conformational equilibrium are thermodynamically linked, it is possible to show that the protein concentration dependence of $\Delta \mathrm{G}^{\circ}$ provides critical information to evaluate the degree of protein aggregation in the formulation. This is the main focus of this paper. 


\section{Materials and Methods}

\section{Proteins and Reagents}

Bovine carbonic anhydrase isozyme II (bCA-II) was purchased as a lyophilized powder from Sigma-Aldrich (St. Louis, MO) (Cat. \# C2522) and was dissolved and further dialyzed into $20 \mathrm{mM}$ succinate $\mathrm{pH}$ 6.0. Cetuximab and Trastuzumab were obtained from LGM Pharma (Nashville, TN). Cetuximab was delivered as a solution of $10 \mathrm{mg} / \mathrm{mL}$ protein in phosphate buffer containing $0.005 \%(\mathrm{v} / \mathrm{v})$ polysorbate, which was removed by dialysis against PBS, pH 7.5 (Roche Diagnostics $\mathrm{GmbH}$, Mannheim, Germany). Trastuzumab was delivered as a solution of $20 \mathrm{mg} / \mathrm{mL}$ protein in $5.42 \mathrm{mM}$ histidine, $\mathrm{pH} 6.0,0.01 \%(\mathrm{v} / \mathrm{v})$ polysorbate 20. Ultrapure urea was from J.T. Baker (Center Valley, PA) and molecular grade guanidinium $\mathrm{HCl}(\mathrm{GdnHCl})$ was from Promega Corporation (Madison, WI). Sodium succinate, histidine, polysorbate 20, trehalose and dextrose were from Sigma-Aldrich (St. Louis, MO).

\section{Isothermal Chemical Denaturation}

All chemical denaturation experiments were carried out using an AVIA ICD 2304 Isothermal Chemical Denaturation System (Norton, MA) using intrinsic protein fluorescence detection. The excitation wavelength was $280 \mathrm{~nm}$ and scans of the emission intensity were recorded between 300 and $500 \mathrm{~nm}$. The buffers used in

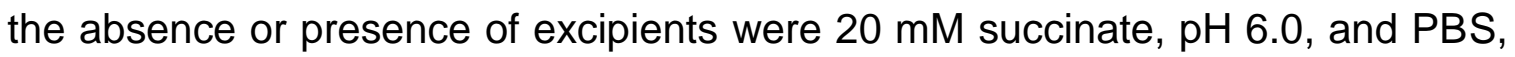
pH 7.5 for bCA-II and cetuximab, respectively. For Trastuzumab, the base buffer was $4.5 \mathrm{mM}$ histidine, $0.009 \%$ polysorbate $20, \mathrm{pH} 6.0$ with $5 \%(\mathrm{w} / \mathrm{v})$ dextrose as 
excipient. The different concentrations of trehalose or L-Arg solutions were automatically prepared by the instrument. For each denaturation experiment, protein, buffer and $10 \mathrm{M}$ urea were dispensed into 36 wells with a linear gradient of urea from 0 to $9 \mathrm{M}$ generated by the instrument. The protein solution is diluted 12.5-fold upon addition to the wells and the protein stocks were therefore prepared at concentrations that accommodate this dilution. In order to allow complete denaturation of cetuximab, the fluorescence intensities were recorded 18 hours after incubation with urea. Trastuzumab denaturations were undertaken using 32 point linear $0-5.52 \mathrm{M} \mathrm{GdnHCl}$ gradients with three hour sample incubation in denaturant. Data processing was performed with the software provided by the instrument and global analysis was performed with the software package Datafit (Oakdale Engineering, Oakdale PA).

\section{Differential Scanning Calorimetry}

Thermal denaturation experiments were performed using a VP-DSC microcalorimeter from MicroCal/Malvern Instruments LLC (Northampton, MA). Solutions of bCA-II were extensively dialyzed against buffer in the absence or presence of $300 \mathrm{mM} \mathrm{L-Arg.} \mathrm{bCA-II} \mathrm{concentration} \mathrm{was} 1.0 \mathrm{mg} / \mathrm{mL}$. The reference cell was filled with dialysis buffer. The buffer was $20 \mathrm{mM}$ succinate, $\mathrm{pH}$ 6.0. The samples were thoroughly degassed before loading of the calorimetric cell ( 0.5 $\mathrm{mL}$ ). Thermal denaturation scans were conducted from 10 to $100^{\circ} \mathrm{C}$ at a rate of $1^{\circ} \mathrm{C} / \mathrm{min}$. 


\section{Results and Discussion}

\section{The Population of Denatured Protein}

Figure 1 illustrates the basic thermodynamic equilibrium model considered in this paper. The native state (red) exists in equilibrium with the denatured state (green) which has a tendency to aggregate in an eventually irreversible manner. The population or fraction of denatured protein $\left(F_{\text {denat }}\right)$ is equal to the total concentration of denatured protein (non-aggregated plus aggregated) divided by the total protein concentration:

$F_{\text {denat }}=\frac{[D]+j\left[D_{j}\right]}{[N]+[D]+j\left[D_{j}\right]}$

Where $\mathrm{j}$ is the average degree of oligomerization of the aggregates. Denatured state aggregation is not a process characterized by well-defined stoichiometries or structures. In our approach, it is represented by an ensemble of diverse aggregates characterized by a mean degree of oligomerization.

In the absence of aggregation, equation 1 reduces to the well-known equation: 


$$
F_{\text {denat }}=\frac{[D]}{[N]+[D]}=\frac{K}{1+K}=\frac{e^{-\frac{\Delta G^{o}}{R T}}}{1+e^{-\frac{\Delta G^{o}}{R T}}}
$$

Which, at any constant temperature, is only a function of the Gibbs energy of stability $\left(\Delta \mathrm{G}^{\circ}\right) . \quad \mathrm{K}$ is the equilibrium constant $\left(K=\frac{[D]}{[N]}\right)$ which is also defined in terms of $\Delta \mathrm{G}^{\circ}$. Equation 2 can be easily generalized to the situation in which a protein exhibits multiple transitions as each transition will be described by an equation similar to Equations 1-2 [10]. In the case in which protein aggregation or self-association is absent, the fraction of denatured protein is independent of protein concentration as it only depends on $\Delta \mathrm{G}^{\circ}$ (right hand side of equation 2). The situation is different if aggregation occurs, since aggregation is a concentration dependent association phenomenon. In general, denatured aggregation will increase the value of $\mathrm{K}$ while native aggregation will decrease its value, facts that are reflected in the magnitude of $\Delta G^{0}$ [11-13]. An experimental test to evaluate the presence or absence of aggregation or self-association is to measure $\Delta \mathrm{G}^{0}$ at different protein concentrations. If $\Delta \mathrm{G}^{\circ}$ is independent of concentration, no aggregation is present. If $\Delta \mathrm{G}^{\circ}$ decreases with concentration denatured aggregation is present, whereas if $\Delta G^{0}$ increases with concentration native state aggregation is present. 
A well-established method to experimentally determine $\Delta G^{\circ}$ consists of measuring the denaturation of the protein with a chemical agent like urea or guanidinium hydrochloride. The Gibbs energy has been experimentally shown to follow a simple linear dependence with the denaturant concentration [14-18]:

$$
\Delta G=\Delta G^{o}-m[\text { denaturant }]
$$

Where $\Delta G^{\circ}$ is the Gibbs energy of stability of the protein at zero denaturant concentration; i.e. the stability of the protein at the experimental temperature and solvent conditions except denaturant. $\mathrm{m}$ is universally referred to as the $\mathrm{m}$-value [15-19]. $\Delta G^{0}$ is the quantity measured by chemical denaturation. The other method to determine $\Delta G^{\circ}$ is differential scanning calorimetry (DSC); however, because the thermal denaturation of many proteins, especially monoclonal antibodies is thermodynamically irreversible, DSC cannot be used to measure their thermodynamic stability $[10,20]$. On the contrary, the chemical denaturation of antibodies is usually reversible under conditions at which temperature denaturation is not, thus allowing $\Delta G^{\circ}$ determination [10]. Chemical denaturation can be performed at different protein concentrations, thus allowing direct measurement of the concentration dependence of $\Delta \mathrm{G}^{\circ}$.

Figure 2 presents two examples of monoclonal antibodies exhibiting opposite $\Delta G^{\circ}$ concentration dependencies. Panel A shows the experimental concentration dependence of $\Delta \mathrm{G}^{\circ}$ for trastuzumab, which is known to readily 
aggregate in the presence of $5 \%$ dextrose [21]. In this case $\Delta G^{\circ}$ increases with protein concentration, as expected for a protein that aggregates in the native

state. In panel $\mathbf{B}$, the experimental concentration dependence of $\Delta G^{\circ}$ for cetuximab is shown. In this case $\Delta \mathrm{G}^{\circ}$ decreases with protein concentration as expected for a protein that aggregates in the denatured state.

\section{Experimental Error in the Determination of $\Delta \mathbf{G}$}

Figure 3 (panel A) shows a set of chemical denaturation curves for cetuximab obtained at zero, 100 and $200 \mathrm{mM}$ trehalose. Trehalose is used as a protein stabilizer in protein therapeutics (e.g. bevacizumab (avastin), ranibizumab (lucentis), trastuzumab (herceptin)). The experiments were performed using urea as the denaturant. For each trehalose concentration, the denaturation experiments were performed in triplicate as shown in the figure. Reversibility of the denaturation transition was confirmed by performing a urea dilution experiment (data not shown). Global analysis of the data yields a $\Delta \mathrm{G}^{\circ}$ at zero trehalose concentration of $8.84 \mathrm{kcal} / \mathrm{mol}$ with a standard deviation of $0.3 \mathrm{kcal} / \mathrm{mol}$. According to the data, trehalose has a stabilizing effect on cetuximab, as indicated by $\Delta \mathrm{G}^{\circ}$ values of 9.43 and $10.0 \mathrm{kcal} / \mathrm{mol}$ at 100 and $200 \mathrm{mM}$ trehalose respectively. It must be noted that an increase in $\Delta \mathrm{G}^{\circ}$ of $1.4 \mathrm{kcal} / \mathrm{mol}$ is equivalent to a ten-fold increase in stability $\left(K=\exp \left(-\Delta G^{\circ} / R T\right)\right)$. This stabilization is reflected in a reduction in the fraction of denatured protein by approximately ten-fold (Figure 3 (panel B)). Figure 3 also shows the errors in the calculated 
fractions that are associated with the errors in the determination of $\Delta \mathrm{G}^{\circ}$. The actual amount of denatured protein can be calculated by multiplying the denatured fraction by the protein concentration in the sample. For this particular experiment performed at $24 \mu \mathrm{g} / \mathrm{mL}$, the results indicate that at zero trehalose, the concentration of denatured protein is $10 \mathrm{pg} / \mathrm{mL}$ and is reduced to about $1 \mathrm{pg} / \mathrm{mL}$ at $200 \mathrm{mM}$ trehalose.

\section{The Concentration Dependence of the Gibbs Energy}

If the denatured state has a tendency to aggregate (Figure 1), the aggregation process can be described in terms of a phenomenological aggregation constant $K_{j}=\frac{\left[D_{j}\right]}{[D]^{j}}$ in which $\mathrm{j}$ is the average degree of oligomerization in the ensemble of aggregates. The initial ensemble of aggregates is assumed to be in equilibrium with the monomeric unfolded state. Irreversibility eventually arises because some aggregates reach a critical size and/or some aggregates become insoluble and fall out of solution. The actual mechanism is immaterial for this analysis. The aggregation process may take months and its actual length or rate has been correlated with some conformational stability parameters, like the temperature denaturation of the protein $[6,22]$. Since chemical denaturation allows determination of the true thermodynamic stability, it also allows quantitative calculation of some aggregation related parameters. For the thermodynamic model presented here, the apparent or observed conformational equilibrium 
constant, $K_{a p p}$, is defined as the ratio of the total concentration of denatured protein (non-aggregated plus aggregated) to the concentration of native protein:

$K_{\text {app }}=\frac{[D]+j\left[D_{j}\right]}{[N]}=K\left(1+j K_{j}[D]^{j-1}\right)$

And, correspondingly, the apparent Gibbs energy becomes:

$\Delta G_{a p p}=\Delta G_{o}^{o}-R T \ln \left(1+j K_{j}[D]^{j-1}\right)$

In equation $5, \Delta G_{o}^{o}$ is equal to the value of the Gibbs energy extrapolated to zero protein concentration (in practice, a concentration low enough at which aggregation is not present). Equation $\mathbf{5}$ indicates that denatured state aggregation will cause the measured Gibbs energy, $\Delta G_{a p p}$, to decrease as a function of protein concentration and that the magnitude of the decrease is proportional to the aggregation constant and the average size of the aggregates. It should be realized that, if the native state were the aggregating species, the concentration dependence of $\Delta \mathrm{G}_{\mathrm{app}}$ will be described by a similar equation to equation 5 except that the minus sign will be replaced by a plus sign. In this 
case, the measured Gibbs energy, $\Delta G_{a p p}$, will increase with protein concentration as shown in Figure 2 panel $\mathbf{A}$.

\section{The Population of Aggregated Protein}

The fraction of protein that is aggregated is calculated according to the following standard equations:

$F_{a g g}=\frac{j\left[D_{j}\right]}{\left([N]+[D]+j\left[D_{j}\right]\right)}=\frac{j K_{j}[D]^{j-1}}{\left(\frac{1}{K}+1+j K_{j}[D]^{j-1}\right)}$

From Equation 5, it is apparent that the term $j K_{j}[D]^{j-1}$ can be expressed solely in terms of the measured $\Delta G$ if its concentration dependence is known:

$F_{a g g}=\frac{\left(e^{-\frac{\Delta \Delta G}{R T}}-1\right)}{\left(e^{\frac{\Delta G_{o}^{O}}{R T}}+1+\left(e^{-\frac{\Delta \Delta G}{R T}}-1\right)\right)}$

Where $\Delta \Delta G=\Delta G_{a p p}-\Delta G_{o}^{o}$. The importance of equation 7 cannot be minimized as it allows estimation of the fraction of aggregated protein without the need to know the free denatured concentration ([D]), the aggregation constant and the average degree of aggregation. In this experimental formalism, the calculation of the aggregated fraction of protein does not require an explicit numerical value for 
$\mathrm{j}$ or $\mathrm{K}_{\mathrm{j}}$ as they can be expressed in terms of $\Delta \Delta \mathrm{G}$. In a similar fashion, the fraction of denatured protein, $F_{\text {denat, }}$ can be expressed as:

$F_{\text {denat }}=\frac{e^{-\frac{\Delta \Delta G}{R T}}}{\left(e^{\frac{\Delta G_{0}^{o}}{R T}}+1+\left(e^{-\frac{\Delta \Delta G}{R T}}-1\right)\right)}$

In a typical protein solution, the vast majority of protein is in the native conformation and therefore the fraction of denatured protein is exceedingly small. For a typical protein in which $\Delta \mathrm{G}$ is on the order of $8 \mathrm{kcal} / \mathrm{mol}$, the denatured fraction is on the order of $10^{-6}$ (Figure 4). Another important quantity is the fraction of denatured protein that is aggregated $\left(F_{\text {agg of }} F_{\text {denat }}\right)$, which is given by the equation:

$F_{\text {agg of } F_{\text {denat }}}=\frac{e^{-\frac{\Delta \Delta G}{R T}}-1}{\left(1+\left(e^{-\frac{\Delta \Delta G}{R T}}-1\right)\right)}$

Figure 4 shows the three fractional populations that can be obtained from the analysis of the data in Figure 2-3 for cetuximab. As can be seen in the figure, the amount of denatured protein and aggregated protein is exceedingly small, as expected in a freshly prepared solution. The denatured fraction amounts to less than $10^{-6}$ but increases to about $10^{-5}$ at the highest concentration measured, as expected by the decrease in $\Delta G$ with concentration. These low amounts are impossible to measure with conventional techniques (e.g. size exclusion 
chromatography, light scattering, etc) but can be accurately calculated from $\Delta \mathrm{G}_{\text {app }}$. The aggregation tendency of the denatured state can be evaluated by knowing the fraction of the denatured protein that is aggregated. In fact, even at the relatively low concentration of $0.18 \mathrm{mg} / \mathrm{mL}$, more than $95 \%$ of the denatured cetuximab is aggregated. A high degree of aggregation of the denatured protein, even if the total amount of denatured protein is low, can be detrimental in the long range. Denatured protein with a high tendency to aggregate will eventually deplete the population of native protein and reduce the viability of the protein solution. In general, a successful formulation strategy should be one that minimizes the amount of denatured protein and simultaneously minimizes the fraction of aggregated denatured protein. From a thermodynamic point of view that strategy means one that is geared to simultaneously maximize $\Delta \mathrm{G}_{\mathrm{app}}$ and minimize its protein concentration dependence. Accordingly, the optimal solution condition is not necessarily the one that only maximizes $\Delta G$ but the one that jointly minimizes the aggregation tendency of the denatured state.

\section{The Mechanism of Action of Solubilizers: The case of L-Arg}

L-arginine (L-Arg) is used often as a chemical agent to reduce the aggregation and improve the solubility of monoclonal antibodies [23-25]. Since denatured proteins aggregate primarily due to the exposure of the hydrophobic core to the solvent, chemicals which bind preferentially to the denatured state and increase its polarity and improve its solubility. The unwanted side effect of any excipient 
that binds preferentially to the denatured state is that it will destabilize the native state. This effect is analogous, but in the reverse, to the known stabilizing effect of ligands that bind to the native state [10]. This is the case of L-Arg which has a guanidinium group similar to the known protein denaturant guanidinium hydrochloride $(\mathrm{GdnHCl})$. The destabilizing effect of L-Arg has recently been shown [25].

Figure 5 shows the effects of L-Arg on cetuximab. The left panel shows the concentration dependence of $\Delta G_{\text {app }}$ in the absence and in the presence of 300mM L-Arg. Two observations are immediately apparent: 1) at low protein concentrations $\Delta G_{\text {app }}$ is smaller in the presence of $L-A r g$, indicative of lower structural stability; and, 2) the concentration dependence of $\Delta \mathrm{G}_{\mathrm{app}}$ is also smaller in the presence of L-Arg indicative of a lower tendency to aggregate. This is the behavior expected from an excipient that preferentially binds to the denatured state and diminishes its tendency to aggregate. At higher protein concentrations, there is an apparent stabilization effect in the presence of L-Arg due to the steady decrease in $\Delta G_{\text {app }}$ when $L-A r g$ is absent. The center panel in Figure 5 depicts the fraction of denatured protein $\left(F_{\text {denat }}\right)$ in the absence and presence of $300 \mathrm{mM} \mathrm{L-Arg} . \mathrm{F}_{\text {denat }}$ is extremely small in both cases $\left(<10^{-5}\right)$ and by itself cannot be used to make a decision regarding the suitability of either formulation. The critical quantity, however, is the amount of denatured protein that is aggregated, which is derived from the concentration dependence of $\Delta \mathrm{G}_{\text {app }}$. In the absence of L-Arg, the fraction of denatured protein that is aggregated is close to $100 \%$ at 
$180 \mathrm{ug} / \mathrm{mL}$ whereas in the presence of L-Arg it is only $20 \%$. According to these experiments, at $180 \mathrm{ug} / \mathrm{mL}$ in the absence of L-Arg about $2 \mathrm{ng} / \mathrm{ml}$ of protein is aggregated whereas in the presence of L-Arg only $0.3 \mathrm{ng} / \mathrm{mL}$ is aggregated. In effect, L-Arg reduces the amount of aggregated protein by a factor of seven.

For some proteins, the effects of L-Arg can also be observed in thermal denaturation experiments. This is shown in Figure 6 where the thermal denaturation of carbonic anhydrase measured by differential scanning calorimetry at a rate of $60^{\circ} \mathrm{C} / \mathrm{h}$ is shown. As indicated in the figure, the addition of $300 \mathrm{mM} \mathrm{L-Arg} \mathrm{lowers} \mathrm{the} \mathrm{denaturation} \mathrm{temperature} \mathrm{by} 5^{\circ} \mathrm{C}$ from 70.5 to $65.5^{\circ} \mathrm{C}$, whereas the aggregation and precipitation temperature increases by a similar amount. In the presence of L-Arg aggregation and precipitation occurs more than 10 degrees above denaturation, whereas in the absence of L-Arg both processes occur almost simultaneously. The DSC results are also consistent with a destabilization of the protein and an increase in the solubility of the denatured protein as it precipitates at much higher temperature.

\section{Conclusions}

Concentration dependence studies of thermodynamic stability provide three critical aggregation parameters, the fraction of denatured protein, the fraction of aggregated protein and the fraction of denatured protein that is aggregated. The magnitude of $\Delta \mathrm{G}_{\mathrm{app}}$ is directly related to the fraction of protein molecules that are 
denatured; and, the concentration dependence of $\Delta G_{a p p}$ is indicative of the degree of aggregation present in the formulation. Both quantities can be assessed immediately after a protein solution is prepared, thus allowing a rapid evaluation of solvent conditions, or alternatively, an evaluation of the protein mutants or clones that exhibit the best stability/aggregation profile. The engineering goal is to identify protein mutations or solvent conditions that maximize $\Delta G$ and simultaneously minimize its concentration dependence.

Ackowledgments: This work was partially supported by a grant from the National Science Foundation MCB-1157506 


\section{References}

[1] U.B. Ericsson, B.M. Hallberg, G.T. DeTitta, N. Dekker, P. Nordlund, Thermofuor-based high-throughput stability optimization of proteins for structural studies, Anal. Biochem, 357 (2006) 289-298.

[2] M. Vedadi, F.H. Niesen, A. Allali-HAssani, O.Y. Fedorov, P.J. Finerty Jr, G.A. Wasney, R. Yeung, C. Arrowsmith, L.J. Ball, H. Berglund, R. Hui, B.D. Marsden, P. Nordlund, M. Sundstrom, J. Weigelt, A.M. Edwards, Chemical screening methods to identify ligands that promote protein stability, protein crystallization and structure determination, Proc. Natl. Acad. Sci. (USA), 103 (2006) 1583515840.

[3] M.A.H. Capelle, R. Gurny, T. Arvinte, High throughput screening of protein formulation stability: Practical considerations, Eurpean J. Pharmaceutics and Biopharmaceutics, 65 (2007) 131-148.

[4] G.A. Senisterra, P.J. Finerty Jr., High throughput methods of assessing protein stability and aggregation, Mol. BioSyst., 5 (2009) 217-223.

[5] Y. Li, C.J. Roberts, Protein Aggregation Pathways, Kinetics, and Thermodynamics, in: W.W.a.C.J. Roberts (Ed.) Aggregation of Therapeutic Proteins, John Wiley \& Sons, Inc.2010, pp. 63-102.

[6] M.L. Brader, T. Estey, S. Bai, R.W. Alston, K.K. Lucas, S. Lantz, P. Landsman,

K.M. Maloney, Examination of Thermal Unfolding and Aggregation Profiles of a Series of Developable Therapeutic Monoclonal Antibodies, Molecular Pharmaceuticals, 12 (2015) 1005-1017. 
[7] R. Lumry, H. Eyring, Conformation Changes in Proteins, J. Phys. Chem., 58 (1954) 110-120.

[8] J.M. Sanchez-Ruiz, Theoretical analysis of Lumry-Eyring models min differential scanning calorimetry, Biophys J, 61 (1992) 921-935.

[9] E.Y. Chi, S. Krishnan, T.W. Randolph, J.F. Carpenter, Physical Stability of Proteins in Aqueous Solution: Mechanism and Driving Forces in Nonnative Protein Aggregation, Pharmaceutical Research, 20 (2003) 1325-1336.

[10] A. Schon, R.K. Brown, B. Hutchins, E. Freire, Ligand binding analysis and screening by chemical denaturation shift, Analytical Biochem, 443 (2013) 52-57.

[11] O. Boudker, M.J. Todd, E. Freire, The structural stability of the cochaperonin GroES, Journal of molecular biology, 272 (1997) 770-779.

[12] M.J. Todd, N. Semo, E. Freire, The structural stability of the HIV-1 protease, Journal of molecular biology, 283 (1998) 475-488.

[13] M.R. Kasimova, S.J. Milstein, E. Freire, The conformational equilibrium of human growth hormone, Journal of molecular biology, 277 (1998) 409-418.

[14] R.F. Greene, Jr., C.N. Pace, Urea and guanidine hydrochloride denaturation of ribonuclease, lysozyme, alpha-chymotrypsin, and beta-lactoglobulin, J. Biol. Chem., 249 (1974) 5388-5393.

[15] C.N. Pace, D.V. Laurents, R.E. Erickson, Urea denaturation of barnase: $\mathrm{pH}$ dependence and characterization of the unfolded state, Biochemistry, 31 (1992) 2728-2734.

[16] M.M. Santoro, D.W. Bolen, Unfolding free energy changes determined by the linear extrapolation method. 1. Unfolding of 
phenylmethanesulfonyl.alpha.chymotrypsin using different denaturants, Biochemistry, 27 (1988) 8063-8068.

[17] D.W. Bolen, M.M. Santoro, Unfolding free energy changes determined by the linear extrapolation method. 2. Incorporation of $d G n-u$ values in a thermodynamic cycle, Biochemistry, 27 (1988) 8069-8074.

[18] J.K. Myers, C.N. Pace, J.M. Scholtz, Denaturant $m$ values and heat capacity changes: Relation to changes in accessible surface area of protein unfolding, Prot. Science, 4 (1995) 2138-2148.

[19] C.N. Pace, Determination and analysis of urea and guanidine hydrochloride denaturation curves, Methods Enzymol, 131 (1986) 266-280.

[20] R.M. Ionescu, J. Vlasak, C. Price, M. Kirchmeier, Contribution of variable domains to the stability of humanized IgG1 monoclonal antibodies, Journal of Pharmaceutical Sciences, 97 (2008) 1414-1426.

[21] B. Demeule, C. Palais, G. Machaidze, R. Gurny, T. Arvinte, New methods allowing the detection of protein aggregates: A case study on trastuzumab, mAbs, 1 (2009) 142-150.

[22] T. Igawa, H. Tsunoda, T. Kuramochi, Z. Sampei, S. Ishii, K. Hattori, Engineering the variable region of therapeutic IgG antibodies, MAbs, 3 (2011) 243-252.

[23] T. Arakawa, D. Eljima, K. Tsumoto, N. Obeyama, Y. Tanaka, Y. Kita, E.N. Timasheff, Suppression of protein interactions by arginine: a proposed mechanism of the arginine effects, Biophys Chem, 127 (2007) 1-8. 
[24] P. Kheddo, M. Tracka, J. Armer, R.J. Dearman, S. Uddin, C.F. van der Walle, A.P. Golovanov, The effect of arginine glutamate on the stability of monoclonal antibodies in solution, International Journal of Pharmaceutics, 473 (2014) 126133.

[25] M. Fukuda, D. Kameoka, T. Torizawa, M. Saitoh, M. Yasutake, Y. Imaeda, A. Kioga, A. Mizutani, Thermodynamic and fluorescence analyses to determine mechanisms of IgG1 stabilization and destabilization by arginine, Pharmaceutical Research, 31 (2014) 992-1001. 


\section{Figure Legends}

Figure 1. Proteins exist in conformational equilibrium between the native (red) and denatured (green) (unfolded or partially unfolded) states. Under some conditions the denatured state may exhibit a tendency to associate or aggregate. This tendency to aggregate affects the conformational equilibrium in a concentration dependent fashion. Analysis of the conformational equilibrium at different protein concentrations allows calculation of different aggregation parameters.

Figure 2. The concentration dependence of $\Delta \mathrm{G}$ for trastuzumab $(4.5 \mathrm{mM}$ histidine, $0.009 \%$ polysorbate $20, \mathrm{pH} 6.0$ plus $5 \%$ dextrose) and cetuximab (10 mM PBS, $\mathrm{pH} 7.5)$. The increase in $\Delta \mathrm{G}$ with concentration observed for trastuzumab is indicative of native state aggregation whereas the decrease in $\Delta G$ with concentration observed for cetuximab is indicative of denatured state aggregation.

Figure 3. Left panel: Urea denaturation of the monoclonal antibody cetuximab in $10 \mathrm{mM}$ PBS and zero, 100 and $200 \mathrm{mM}$ of trehalose. Each experiment was performed in triplicate and the effect of trehalose evaluated by global analysis of the nine independent experiments shown in the graph. The average residual of the fit was $7.39 \times 10^{-4}$ and the coefficient of multiple determination $\left(\mathrm{R}^{2}\right)$ was 0.9934 . Right panel: The fraction of denatured 
protein as a function of trehalose concentration. The error bars are derived from the errors in the determination of $\Delta \mathrm{G}$ (see text for details).

Figure 4. Analysis of the concentration dependence of $\Delta \mathrm{G}$ yields three important quantities: the fraction of denatured protein (left panel), the total fraction of aggregated protein (center panel) and the fraction of denatured protein that is aggregated.

Figure 5. The effects of L-Arg on the aggregation profile of cetuximab in $10 \mathrm{mM}$ PBS, pH 7.5. The left panel shows the concentration dependence of $\Delta \mathrm{G}$ in the absence (blue lines) and in the presence (red lines) of 300mM L-Arg. The center panel shows the concentration dependence of the fraction of denatured protein and the right panel, the fraction of denatured protein that is aggregated in the absence and in the presence of $300 \mathrm{mM} \mathrm{L-Arg.}$ These figures illustrate the suppression of denatured state aggregation by L-Arg.

Figure 6. The thermal denaturation of carbonic anhydrase in $20 \mathrm{mM}$ succinate, $\mathrm{pH} 6.0$, in the absence (blue) and in the presence (red) of $300 \mathrm{mM} \mathrm{L-Arg.}$ The presence of L-Arg destabilizes the protein (lower $T_{m}$ ) but shifts to higher temperatures the aggregation and precipitation of the protein $\left(T_{\text {agg }}\right)$. 
Figure 1

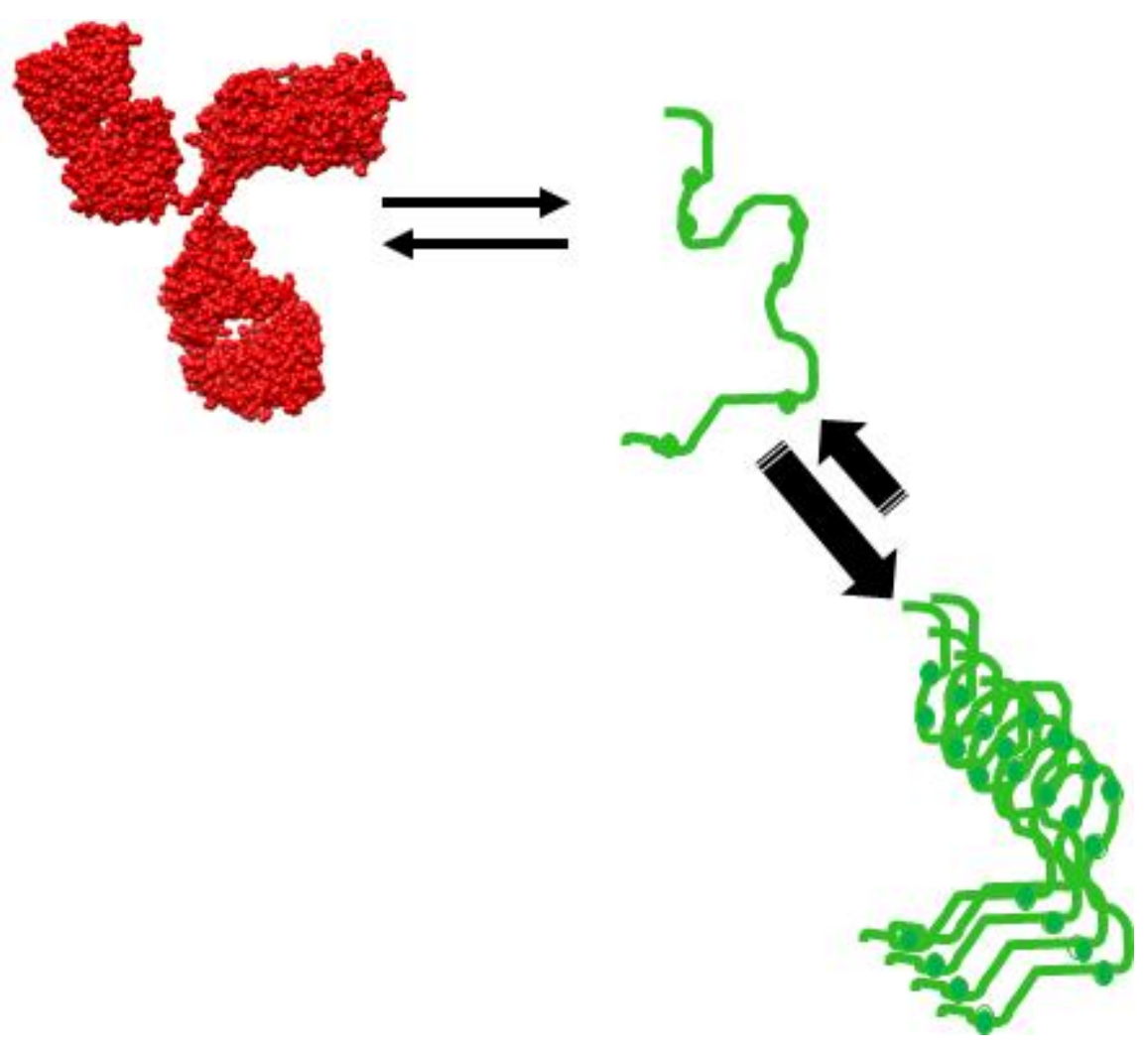


Figure 2
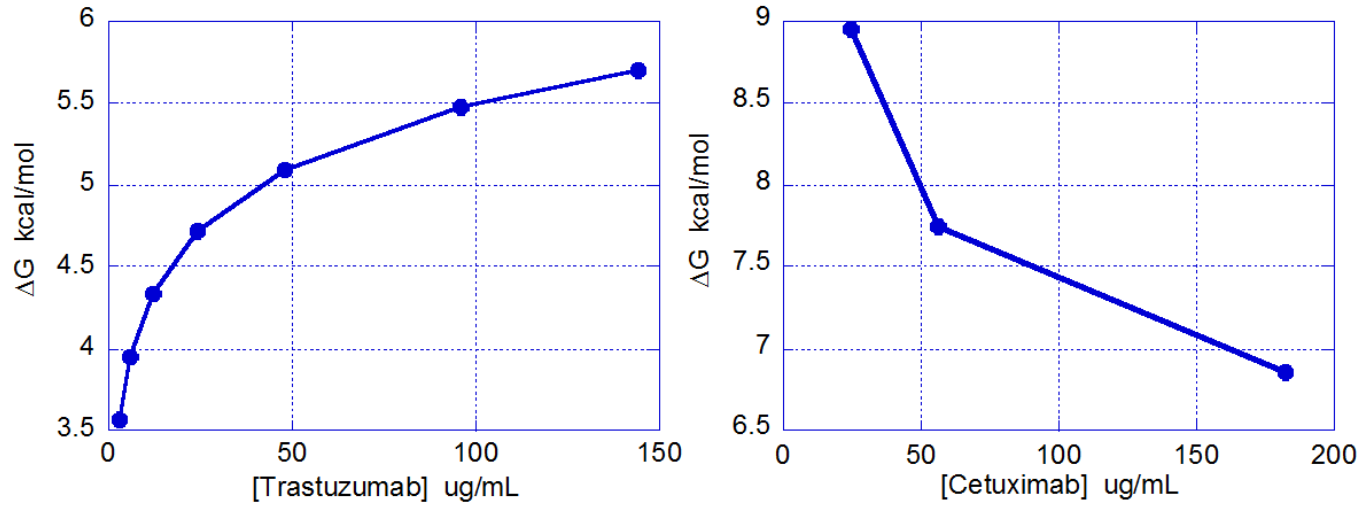
Figure 3
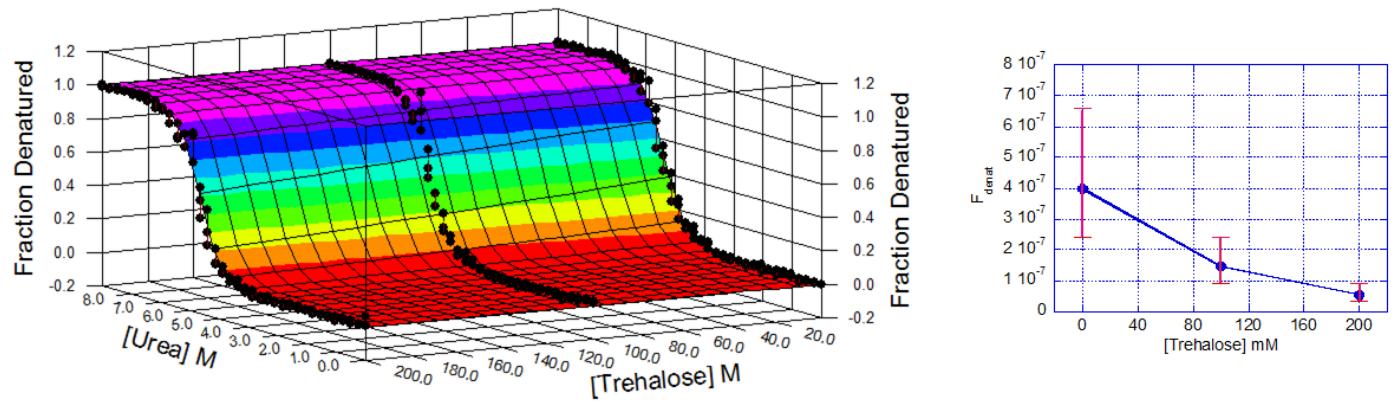
Figure 4
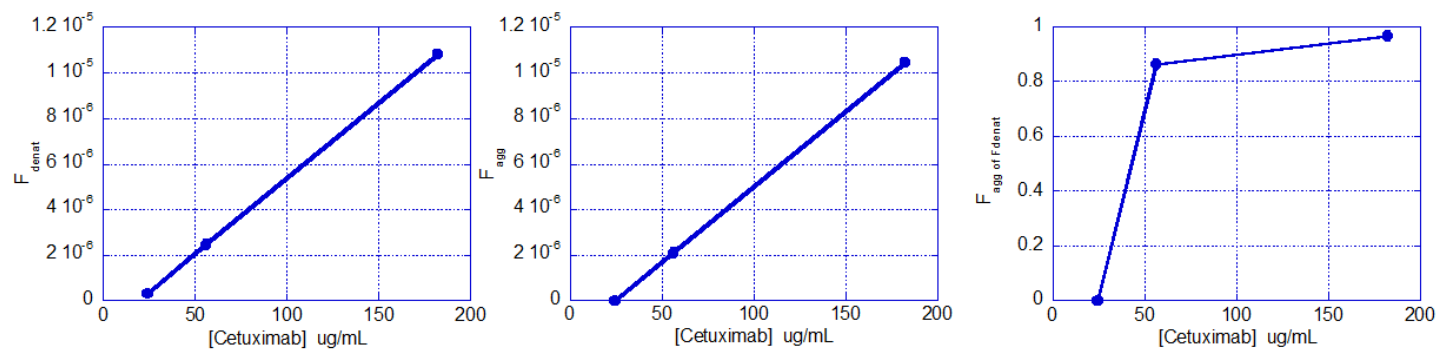
Figure 5
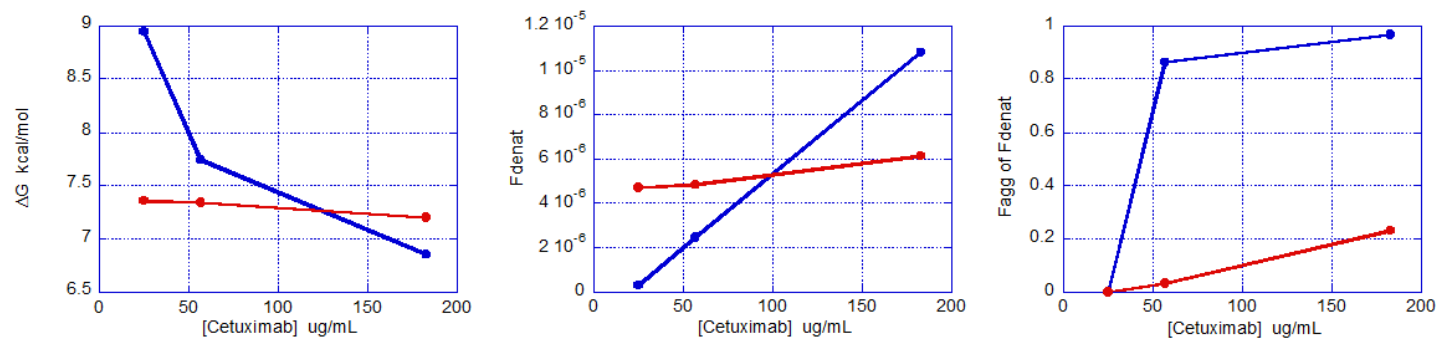
Figure 6

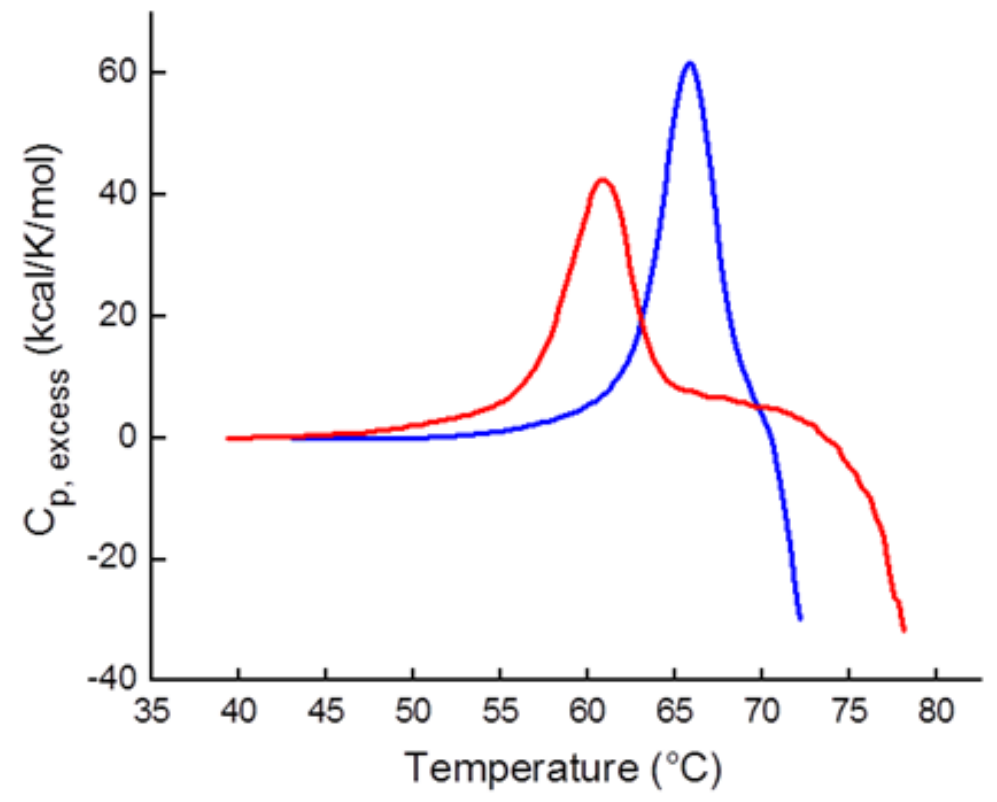

